\title{
PULMONARY-RENAL SYNDROME IN A CHILD WITH SYSTEMIC LUPUS ERYTHEMATOSUS: CASE REPORT
}

Tatiane Comunello ${ }^{1, \star}$, Tiago Osternack Malucelli ${ }^{1}$, Irides Aparecida Cavalari ${ }^{1}$, Guilherme Welter Wendt ${ }^{1}$, Isadora Nunes Ferreira ${ }^{1}$

1.Universidade Estadual do Oeste do Paraná, Francisco Beltrão (PR), Brazil.

*Corresponding author: tati_comunello@hotmail.com

\section{BACKGROUND}

Pulmonary-renal syndrome is a life-threatening condition that consists of the association between diffuse alveolar hemorrhage and rapidly progressive glomerulonephritis. It is a rare complication in autoimmune diseases, such as systemic lupus erythematosus. Diffuse alveolar hemorrhage is rare in patients with systemic lupus erythematosus, with death rates of up to $92 \%$ of cases.

\section{CASE REPORT}

This is a 13-year-old female patient diagnosed with systemic lupus erythematosus presenting with hemoptysis, cough, dyspnea at rest, chest pain, low hematocrit, bilateral diffuse alveolar infiltrate (Fig. 1), hypoxemic respiratory failure, anasarca, oliguria, malar erythema and progressive deterioration of the general condition. She was treated in an emergency room with a pulsed intravenous of methylprednisolone ( $1 \mathrm{~g} /$ day) and underwent intensive care, when received two more pulsed intravenous dose of methylprednisolone $(1 \mathrm{~g} /$ day). She reached a favorable outcome after diagnosis of pulmonary-renal syndrome, because the immunosuppression and appropriated management, intensive care unit (ICU) admission, hemodialysis, blood transfusion and antibiotic therapy.

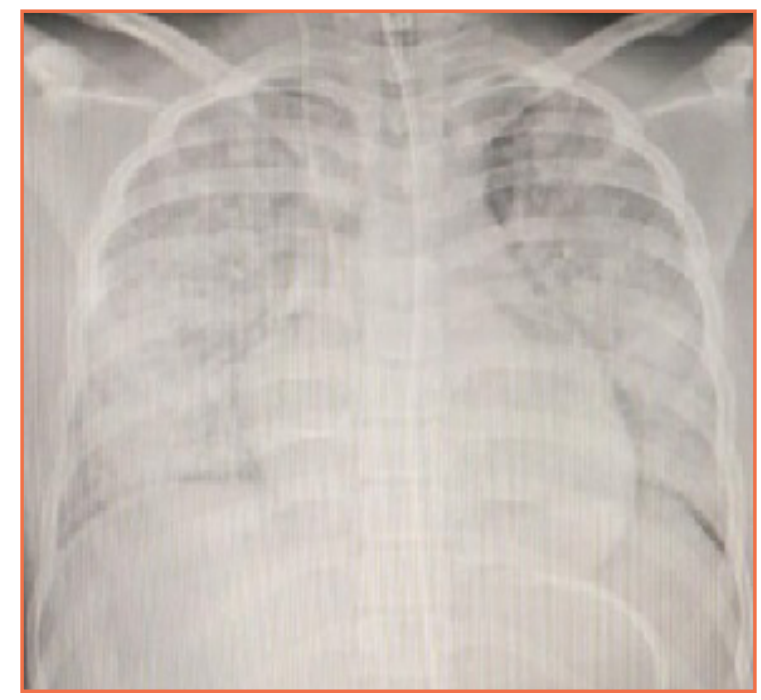

Source: medical record (image authorized by the patient's guardians).

Figure 1. Chest radiography with bilateral diffuse pulmonary opacities, some tending to consolidate, evidence of diffuse alveolar hemorrhage.

\section{CONCLUSION}

Pulmonary-renal syndrome in the context of systemic lupus erythematosus is a rare and severe syndrome, especially in childhood. Their identification, clinical suspicion and early intensive treatment increase the chance of a better prognosis. Without proper management, a complication leads to renal and/or respiratory failure and death. In this case, a resolution of the patient's condition was possible due to rapid diagnosis and appropriate treatment. 